\title{
Xenopus Tbx6 mediates posterior patterning via activation of Wnt and FGF signalling
}

Xin Lou ${ }^{1,2, *}$, Panfeng Fang ${ }^{1,2, *}$, Shuangwei $\mathrm{Li}^{1,2}$, Rui-Ying Hu ${ }^{1,2}$, Klaus-Michael Kuerner ${ }^{3}$, Herbert Steinbeisser ${ }^{3}$, Xiaoyan Ding ${ }^{1,2}$

${ }^{1}$ Laboratory of Molecular Cell Biology, Institute of Biochemistry and Cell Biology, ${ }^{2}$ Shanghai Institutes for Biological Sciences, Chinese Academy of Sciences, Graduate School of the Chinese Academy of Sciences, 320 Yue Yang Road, Shanghai 200031, China; ${ }^{3}$ Institute of Human Genetics, University of Heidelberg, Im Neuenheimer Feld 366, Heidelberg 69120, Germany

In vertebrates, the patterning of anterior-posterior (AP) axis is a fundamental process during embryogenesis. Wnt and FGF signalling pathways play important roles in regulating the patterning of embryo AP axis. Mouse Tbx6 encodes a transcription factor that has been demonstrated to be involved in the specification of the posterior tissue in mouse embryonic body. Here, we prove that morpholino-induced knockdown of XTbx6 impairs posterior development, indicating the requirement of XTbx6 in this process. Meanwhile, gain of XTbx6 function is sufficient to induce ectopic posterior structures in Xenopus embryos. Furthermore, XTbx6 activates the expression of Xwnt 8 and FGF8, which are two mediators of posterior development, suggesting a mechanism by which XTbx6 modulates posterior patterning via Wnt and FGF signalling pathway activation.

Cell Research (2006) 16:771-779. doi: 10.1038/sj.cr.7310093; published online 5 Sep 2006

Keywords: posterior patterning, $X T b x 6$, Wnt, FGF

\section{Introduction}

All vertebrate embryos develop along a well-patterned anterior-posterior (AP) axis. Distinct signalling centers, known as organizers, have been identified for patterning either the anterior part of the body (head) or the posterior part (trunk/tail) [1]. In Xenopus, the posterior body develops from tailbud, which contains pluripotent mesenchymal cells [2]. It has been proved that tailbud tissues function as a tail organizer and several signalling pathways are engaged in Xenopus posterior development [3-6].

The Wnt/ $\beta$-catenin signalling pathway functions in pos-

\footnotetext{
*These two authors contributed equally to this work.

Current address: Panfeng Fang, Department of Molecular, Cellular and Developmental Biology, Yale University, 219 Prospect Street, New Haven, CT 06520, USA

Correspondence: Xiaoyan Ding

Tel: +86-21-54921411;

E-mail: xyding@sunm.shcnc.ac.cn

Received, 5 Jul 2006; revised, 8 Aug 2006; accepted, 10 Aug 2006; published online 5 Sep 2006
}

terior development as an essential and conserved activity module. Several Wnt family members such as wnt $3 a$, wnt 8 and $w n t 5$ are expressed in the posterior part of the vertebrate embryo and active $\mathrm{Wnt} / \beta$-catenin signalling promotes the formation of posterior structures [7-9]. Mice lacking the wnt3 gene do not develop posterior structures, indicating that $\mathrm{Wnt} / \beta$-catenin signalling is required in this patterning process $[7,10]$. There are also extensive crosstalks between signalling cascades. During posterior body patterning, Wnt signalling pathway interacts with Notch signalling and retinoic acid (RA), which has an activity gradient along the A-P axis [11-13].

Another group of potent posteriorizing molecules during vertebrate AP patterning are FGFs. FGFs are able to convert anterior neural tissue to more posterior fates, and inhibition of FGF signalling causes strong posterior defects in Xenopus $[14,15]$. Experiments in mouse, chick and Xenopus strongly suggest that FGF signalling regulates early AP patterning via the modulation of Hox gene expression [16]. The fact that FGFs function as posteriorizing factors was also demonstrated in chicken neural tube formation, 
in which they mediate the maintenance of neural stem cells at Hensen's node. Therefore, FGFs might prolong the time window during which cells are exposed to other posteriorizing factors, such as RA or Wnts. [17]. However, despite the importance of Wnt and FGF proteins in posterior development, little is known about their transcriptional regulation.

Tbx6 encodes a T-box transcription factor expressed in mesoderm tissues fated to form the posterior part of the embryonic body. Tbx6 mutant mice show an enlarged tailbud mainly composed of undifferentiated mesenchymal cells, and three neural tubes form at the expense of the posterior somites [18]. These data suggest that Tbx6 plays an essential role in posterior patterning through transcriptional regulation of its targets. In this study, we show in Xenopus, antisense morpholino oligonucleotide-mediated knockdown of $X T b x 6$ leads to disruption of the posterior structures. Gain of $X T b x 6$ induces well-patterned posterior structures in embryos. $X w n t 8$ and $F G F 8$ were identified as targets of $X T b x 6$, supporting the interpretation that $X T b x 6$ exerts its function in posterior development by modulating Wnt and FGF signalling.

\section{Materials and Methods}

\section{Xenopus embryo manipulation and microinjection}

Xenopus laevis fertilized eggs were obtained, dejellied and cultured as described previously [19], staged according to Nieuwkoop and Faber [20]. In tailbud cutting experiment (Figure 1), the embryos were manipulated in $1 \times$ MBS and the tailbuds were cultured in $1 \times$ MBS. In Figure 1, XTbx6Mo or XTbx6Mo mixed with XTbx6 mRNA or control Mo was injected into ventral marginal zone at two-cell stage or four-cell stage. XTbx6 plasmid was injected into dorsal animal pole at two-cell stage or four-cell stage. For co-immunoprecipitation, XTbx6 mRNA mixed with Lef-1 plasmid or XTbx6Mo mixed with Lef-1 plasmid was injected into marginal zone. For western blot, XTbx6 mRNA or XTbx6Mo was injected into marginal zone. The final concentration for injection is XTbx6 mRNA $400 \mathrm{ng} / \mathrm{ml}$, Lef-1-Engrailed mRNA $400 \mathrm{ng} / \mathrm{ml}$, XFD mRNA $400 \mathrm{ng} / \mathrm{ml}, X T b x 6$ plasmid $70 \mathrm{ng} / \mathrm{ml}$ and Lef-1-HA plasmid 70ng/ml. Each embryo was loaded 3-5 nl mRNA or DNA.

\section{Morpholino}

The morpholino antisense oligonucleotide designed to act against XTbx6 was GAG CTC AGA GTG GTA CAT GGC TGC TG, and its control was GTG CAG CCA TGT ACC ACT CTG AGC TG. Morpholino was stored on $-80{ }^{\circ} \mathrm{C}$ and diluted in TE to the final concentration of $0.2 \mathrm{nM} / \mathrm{ml}$ for using.

The in vitro transcription/translation of XTbx6Mo specificity test was performed according to Transcription and Translation kit (TNT)coupled reticulocyte lysate system (Promega, L4610).

Constructs and in vitro transcription of RNA and in situ hybridization

$X T b x 6$ coding sequence was cloned into plasmid pCS2+ with HindIII (5') and XbaI (3'); for preparing XTbx6 mRNA, the pCS2-
XTbx6 was linearized with NotI and transcribed with SP6 RNA polymerase [19]. Xbra coding sequence was cloned into plasmid pCS2+ with $X b a \mathrm{I}\left(5^{\prime}\right)$ and EcoRI (3'); for preparing Xbra probe, the plasmid was linearized with NotI and transcribed with SP6 RNA polymerase. For preparing Xwnt $3 a$ probe, a part of Xwnt $3 a$ cDNA sequence was cloned into plasmid pCS2+ with EcoRI (5') and XhoI $\left(3^{\prime}\right)$, and the primers for Xwnt $3 a$ probe were GCA GAA TTC AGA TGG GCT GCT TTG GAT AT (forward) and CAA ATT CTC GAG ACA CCA TG (reverse). The pCS2+Xwnt3a-probe plasmid was linearized with NotI and transcribed with SP6 RNA polymerase. The other templates for probe synthesis were as follows: pCS2-FGF8 was linearized with XhoI and transcribed with T7 RNA polymerase [21], pCS2-XLDeltaI was linearized with EcoRV and transcribed with T7 RNA polymerase [22], pCS107-Xwnt8-HA was linearized with XhoI and transcribed with T7 RNA polymerase (from Wylie-Heasman lab), pGS1-HoxB9 was linearized with EcoRI and transcribed with T7 RNA polymerase [23], pBluescript1-Shh was linearized with BamHI and transcribed with T3 RNA polymerase [24], Lef-1Engrailed was linearized with $K p n I$ and transcribed with SP6 RNA polymerase and XFD was linearized with EcoRI and transcribed with SP6 RNA polymerase [14].

The templates preparation for mRNA or probes were performed as described previously [19] (Ambion, Roche ). In situ hybridizations were performed as described [25].

\section{Immunohistochemical reaction}

Whole-mount immunohistochemical reactions were performed by using Xen1 [26] (Developmental Studies Hybridoma Bank, University of Iowa) or 12/101 [27] (Developmental Studies Hybridoma Bank, University of Iowa) antibody as described previously [28].

\section{Animal cap reverse-transcription $P C R$}

XTbx6 mRNA was injected into animal poles of frog embryos at either two-cell stage or four-cell stage. Animal caps were dissected at stage 8 , cultured in $1 \times$ MBS and harvested at either stage 11 or stage 11.5. RNA isolation and reverse transcription were performed as described [19] (Invitrogen). The optimal programs were determined by titration. ODC $\left(55^{\circ} \mathrm{C}, 25\right.$ cycles $), \mathrm{FGF} 8\left(55^{\circ} \mathrm{C}, 28\right.$ cycles $)$ and Xwnt8 $\left(55^{\circ} \mathrm{C}, 28\right.$ cycles $)$. Primers for ODC were CAG CTA GCT GTG GTG TGG (forward) and CAA CAT GGA AAC TCA CAC C (reverse) [29]. Primers for FGF8 were CTG GTG ACC GAC CAA CTA AG (forward) and ACC AGC CTT CGT ACT TGA CA (reverse) [30]. Primes for $X w n t 8$ were TAT CTG GAA GTT GCA GCA TAC A (forward) and GCA GGC ACT CTC GTC CCT CTG $\mathrm{T}$ (reverse) [31].

\section{Co-immunoprecipitation and Western blot}

HA-tagged Lef-1 constructs, alone or together with XTbx6MO or XTbx6 mRNA, were injected into the DMZ, and the embryos were then cultured in $0.1 \times$ MMR and collected until stage 20 . The embryos were lysed in lysis buffer containing $50 \mathrm{mM}$ Tris- $\mathrm{Cl}(\mathrm{pH}$ $8.0), 150 \mathrm{mM} \mathrm{NaCl}, 10 \%$ glycerol, $1 \%$ Nonidet P-40 and cocktail protease inhibitors (Bio Basics), stored on ice for $15 \mathrm{~min}$ and mixed with $3 / 5$ volume FREON. One-tenth of the supernatant after $13000 \mathrm{rpm}$. centrifuge was saved for loading control and the rest was incubated with protein-A agarose (Santa Cruz) and anti-HA monoclonal antibody (dilution 1:1000) (Sigma, Cat. No. H9658) with mild shaking at $4{ }^{\circ} \mathrm{C}$ for $2 \mathrm{~h}$. The protein-A agarose beads was washed three times and then boiled in $1 \times$ protein loading buffer and subjected to western blot. 

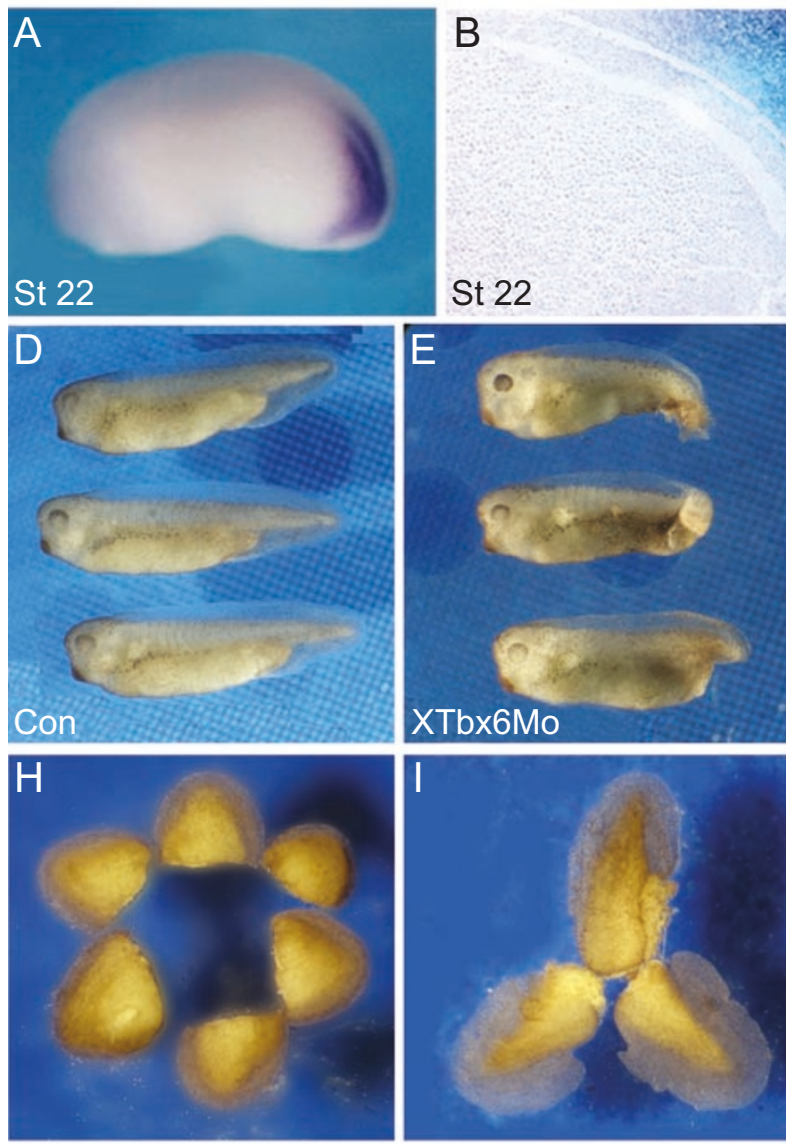

Oh

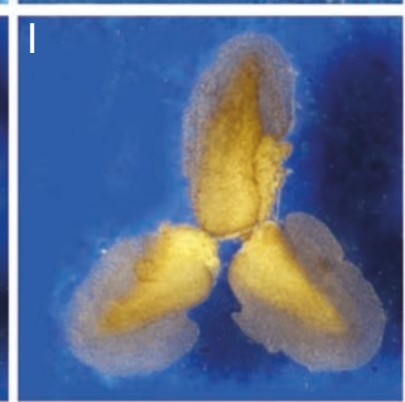

$24 \mathrm{~h}$
C AGCAGCCATGTACCACTCTGAGCTC GTCGTCGGTACATGGTGAGACTCGAG
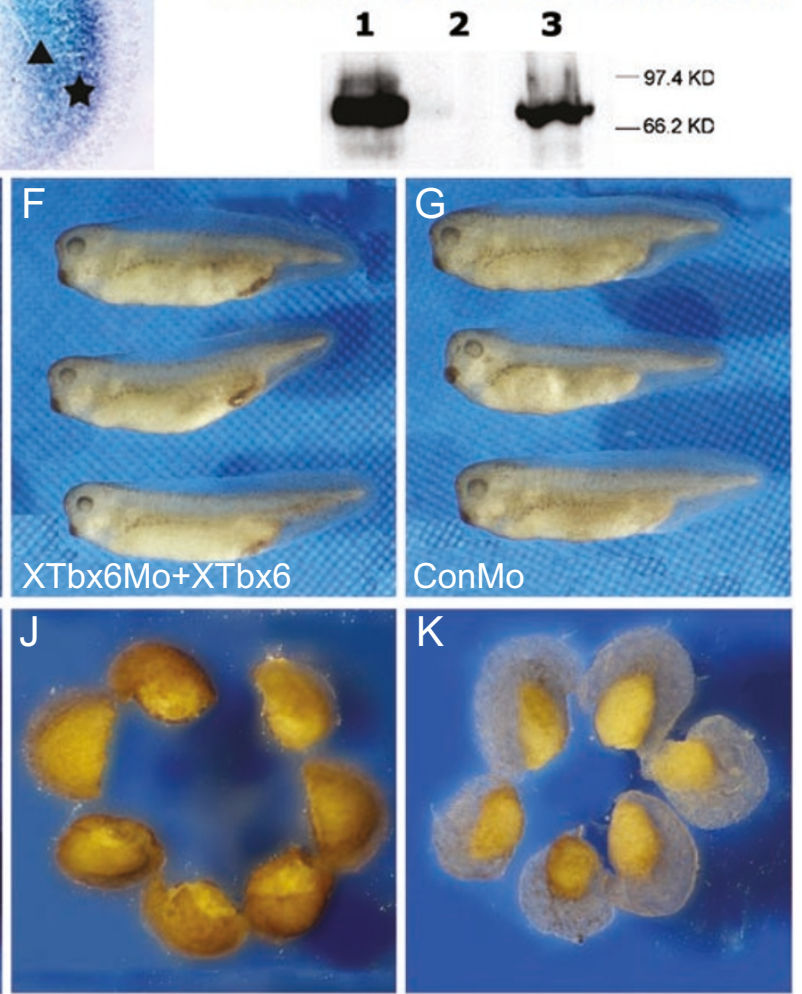

Oh

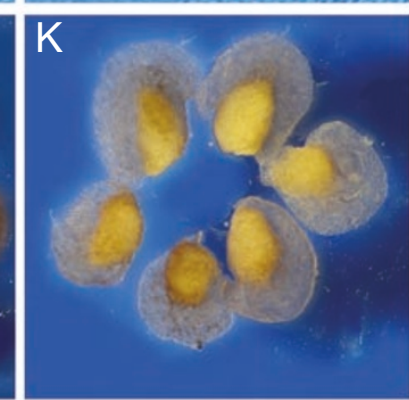

$24 \mathrm{~h}$

Control

XTbx6Mo injection

Figure 1 Reduction of $X T b x 6$ disrupts the posterior structure. (A and B) XTbx 6 was expressed in the chordoneural hinge (triangle) and the posterior wall (asterisk). (A) Lateral view, head to the left. (B) Sagittal midline section of (A). (C) Schematic diagram illustrating the morpholino oligonucleotides (XTbx6Mo) repressing XTbx6 mRNA translation and the specificity test of XTbx6Mo. The specificity of XTbx6Mo is demonstrated by TNT assays. XTbx6 protein is synthesized when the XTbx6-myc construct was incubated with an irrelevant morpholino (lane 1), but was abolished in the presence of XTbx6Mo (lane 2). XTbx6Mo could not abolish the translation of XTbx6-s-Myc, which is similar to XTbx6-Myc but lacks the XTbx6Mo binding sites (lane 3). (D) Control embryos. (E) XTbx6Mo injected into the ventral marginal zone at four-cell stage could induce disruption of the posterior structure. (F) Co-injecting XTbx6Mo with XTbx6 mRNA could rescue the phenotype. (G) Control Mo injection has no obvious phenotype. (D-G) Stage 36, lateral view, with head to the left. (H-K) Bisected tailbuds from stage 23 embryos were cultured for 24 h. (H) Control tailbuds were cultured for 0 h. (I) Control tailbuds could autonomously produce a well-patterned posterior structure. (J) XTbx6Mo-loaded tailbuds were cultured for 0 h. (K) Tailbuds loaded with XTbx6Mo failed to elongate and could not display the morphological characteristics of tails.

Western blot was performed as described [32]. Polyclonal antibody P14L was used for visualizing $\beta$-catenin (dilution 1:2 000) and anti-HA monoclonal antibody (dilution 1:10 000) was used for HA-tagged Lef-1. Both ERK (Cell Signaling Technology, Cat. No. 9102) and phospho-ERK antibody (Cell Signaling Technology, Cat. No. 9101) (dilution 1:2000) were used according to the manufacturer's instructions. For loading control, 1/5 of the loading volume for detection of ERK and phosphor-ERK was used for monoclonal anti- $\beta$-actin antibody (Sigma, dilution 1:10 000).

\section{Results}

Knockdown of XTbx6 function disrupts the posterior development

The previous study $[33,34]$ reported that $X T b x 6$ mRNA is expressed in the mesoderm of embryos from gastrulation onward, especially in cells destined to form posterior mesoderm structures. We performed a more detailed analysis 
of $X T b x 6$ expression in tailbud stage embryos, and found that $X T b x 6$ mRNA is present in the chordoneural hinge and the posterior wall (Figure 1A and 1B), which are both important components of the tail organizer [3]. This distinct expression pattern strongly suggests that $X T b x 6$ is implicated in posterior pattern formation during Xenopus embryogenesis.

In order to investigate the potential role of XTbx 6 in posterior patterning, we performed the knockdown experiment by targeting $X T b x 6$ translation with a morpholino oligonucleotide (XTbx6Mo), which is complementary to the initial codons of $X T b \times 6$ mRNA (Figure 1C). The specificity of XTbx6Mo was examined in the in vitro transcription and translation system. As shown in Figure 1C, the $67 \mathrm{kDa}$ XTbx6-Myc protein was synthesized when control morpholino (a mutant version of XTbx6 morpholino) was added into the in vitro transcription and translation system (Figure $1 \mathrm{C}$, lane 1), but the $67 \mathrm{kDa}$ band was abolished when XTbx6Mo was added (Figure 1C, lane 2). However, XTbx6Mo could not abolish the translation of XTbx6-s-Myc, which has a sequence similar to XTbx6-Myc but just lacks the XTbx6Mo binding sites (Figure 1C, lane 3). XTbx6Mo was injected at four-cell stage into the ventral marginal zone, which will later form the posterior mesoderm, and embryos were cultured to NF stage 35 . XTbx6Mo-injected embryos displayed severe posterior defects (47/50), such as shortened tailbud, distorted posterior body axis and disorganized somites (Figure 1E). In contrast, embryos injected with control Mo did not display any defects (Figure 1G). Co-injection of XTbx6Mo with XTbx6 mRNA completely rescued the defects (Figure 1F).

Xenopus tailbuds isolated and cultured in vitro will continue to grow, forming a tail that is morphologically identical to the endogenous one with myotomes, notochord, neural tube and fin [3]. To further test whether XTbx6 is required for patterning posterior body, we dissected the tailbud region including $X T b x 6$-positive tissues at stage 23 , and cultured them until stage 35 . While control tailbuds elongated and formed tails (Figure 1H and 1I), XTbx6Mo-injected explants did not elongate and displayed no morphological changes, except the development of fin (Figure $1 \mathrm{~J}$ and $1 \mathrm{~K}$ ). Taken together, these results indicate that $X T b x 6$ is required for posterior body patterning of Xenopus embryo.

Ectopic expression of XTbx6 induces axis structures with the posterior characteristics

To further investigate the biological significance of $X T b x 6$ in posterior patterning, gain-of-function experiments were performed in Xenopus embryos. When XTbx6 was ectopically expressed after midblastula transition (MBT) by injection of $X T b x 6$ expression plasmid into the dorsal animal blastomeres (Figure 2A), ectopic structures were induced (34/73) (Figure 2B). These ectopic structures had well-developed fins (Figure 2B), correctly distributed melanocytes (Figure 2C) and a proctodeum (Figure 2D and $2 \mathrm{E}$ ), which are all morphological characteristics of posterior body.

Immunohistochemical staining and in situ hybridization were performed to define the tissues within the XTbx6-in-
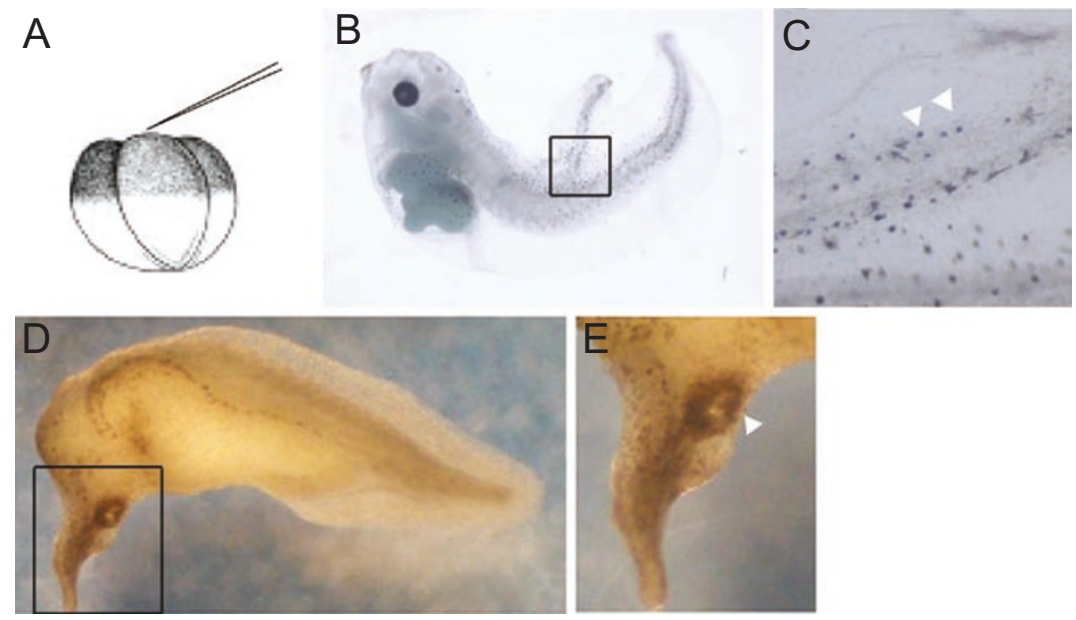

Figure 2 Ectopic expression of XTbx6 induces the posterior structure. (A) A schematic representation of XTbx6 plasmid injected into the dorsal animal pole at four-cell stage. (B) The posterior structure was induced. (C-E) This structure has perfect morphological characteristics such as well-developed fin, melanocytes with the right configuration and distribution (C), and a fully-grown proctodeum (D and E). (B) and (E) Lateral view, head to the left. (C) Magnification of the boxed area in (B). (E) Magnification of the boxed area in (D). 
duced structures. Well-developed neural tubes, marked with the neural specific antibody Xen1, were observed (Figure $3 \mathrm{~A}$ and $3 \mathrm{~B}$ ). Notochord identified by the expression of $S h h$ positioned underneath the neural tube, and the Shh signal was also visible in the floor plate of ectopic neural tube (Figure 3C). Using the muscle-specific antibody 12/101, we found very strong staining in XTbx6-induced structures. In some cases, 12/101-positive cells showed typical metameric arrangement (Figure 3D and 3E), indicating the differentiation of somites. Our results clearly show that the structures induced after $X T b x 6$ ectopic expression contain muscle, notochord and neural tissue. The spatial arrangement of these tissues in the ectopic structures is identical to the endogenous pattern. Gain of XTbx6 function is sufficient to induce well-patterned posterior structures.

Since Wnt and FGF signalling pathways are essential for posterior body patterning, we asked whether these pathways are also involved in the formation of the ectopic structure. The $X T b x 6$-induced posterior structures expressed the Wnt ligands $X w n t 8$ (Figure 3F), Xwnt3a (Figure 3G) as well as the Wnt pathway downstream target XDeltal (Figure 3H) in a spatial pattern similar to that in endogenous development. The same was true for $F G F 8$ (Figure 3I) and the FGF target genes Xbra (Figure 3J) and HoxB9 (Figure $3 \mathrm{~K})$. These data argue that the $X T b x 6$-induced structures are formed by the same or similar mechanisms as those in endogenous embryos.

\section{XTbx6 mediates posterior patterning via Wnt and FGF signalling activation}

In the XTbx6-induced posterior body structure, Xwnt8, $X w n t 3 a, X D e l t a I, F G F 8$ and Xbra were expressed (Figure 3 ). It suggested the possibility that $X T b x 6$ might function in transcriptional regulation of some molecules controlling posterior patterning. Therefore, we raise a hypothesis that XTbx6 might mediate posterior patterning through activating the Wnt and FGF signalling pathways.

Animal cap assay was used to elucidate whether $X T b x 6$ induces $X w n t 8, X w n t 3 a$ and $F G F 8$ expression. We found $X T b x 6$ mRNA induces the expression of $X w n t 8$ and $F G F 8$ (Figure 4F), but $X w n t 3 a$ was not induced in the animal cap assay (data not shown). Endogenous Xwnt 8 and $F G F 8$ expression were downregulated when XTbx6Mo was injected
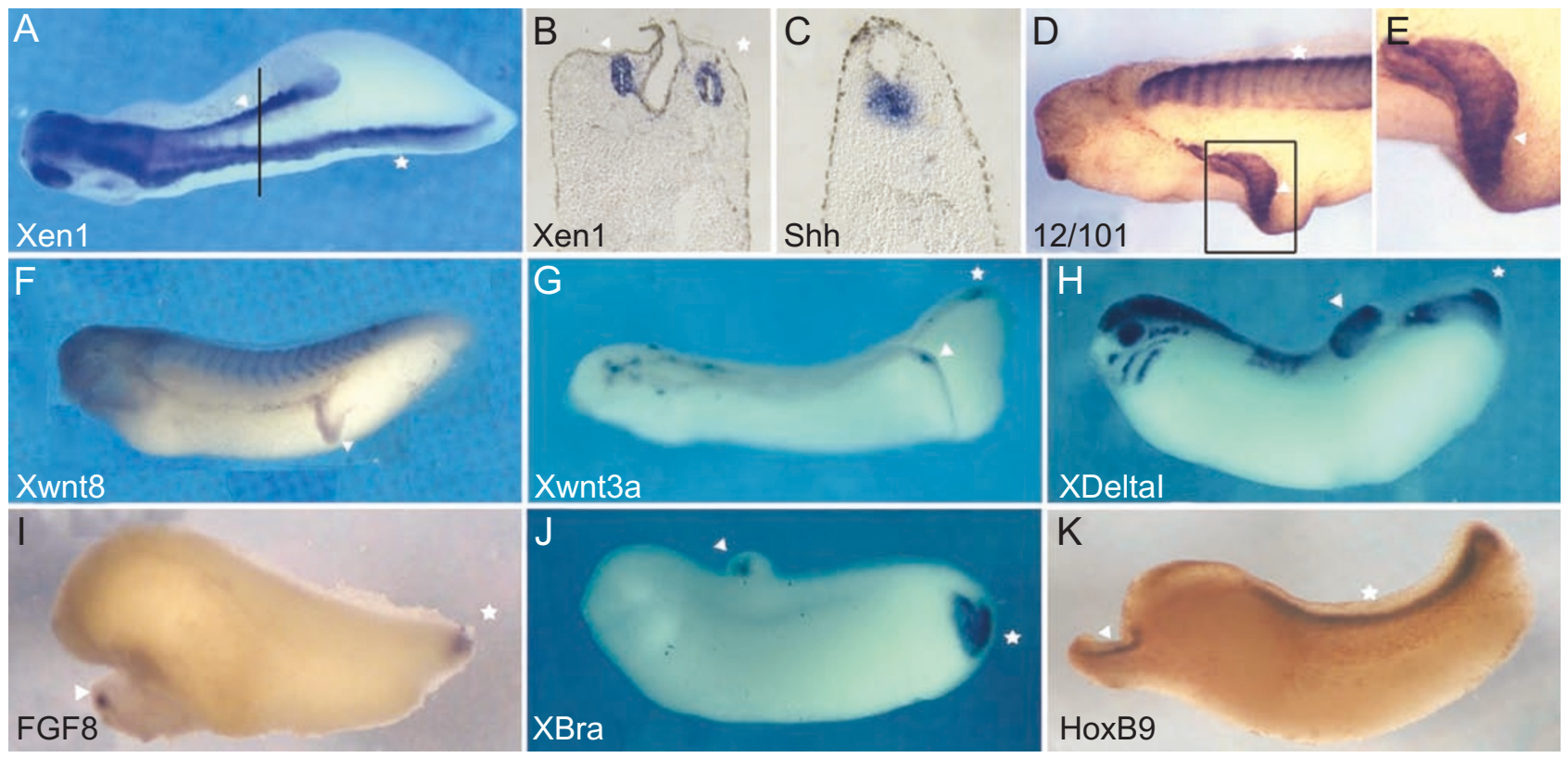

Figure 3 The posterior structures induced after XTbx6 ectopic expression contain muscle, notochord and neural tissue, which were perfectly patterned. (A-E) In the ectopic posterior structure, Xen1 (neural tube marker) (A and B), shh (notochord marker, also expressed in the floor plate of neural tube) (C) and 12/101 (D and E) (myotome marker) stainings are all positive and form a perfect pattern that is the same as the normal embryo. $(\mathbf{F}-\mathbf{H}) X w n t 8(\mathbf{F})$, Xwnt3a $(\mathbf{G})$ and XDeltaI $(\mathbf{H})$ are also expressed in the structure and represent a correct pattern as in the tailbud. (I-K) $F G F 8(\mathbf{I}), X b r a(\mathbf{J})$ and $H o X B 9(\mathbf{K})$ are expressed in the ectopic structure and represent a correct pattern as in the tailbud. (A) Dorsal view, head to left. (D, F-K) Lateral view, head to the left. (B) Cross-section of (A) as the line indicated. (C) Cross-section of an ectopic structure. (E) Magnification of the boxed area in (D). The white trigons indicate signal in ectopic structure, and the white pentacles indicate signal in endogenous tissue. 

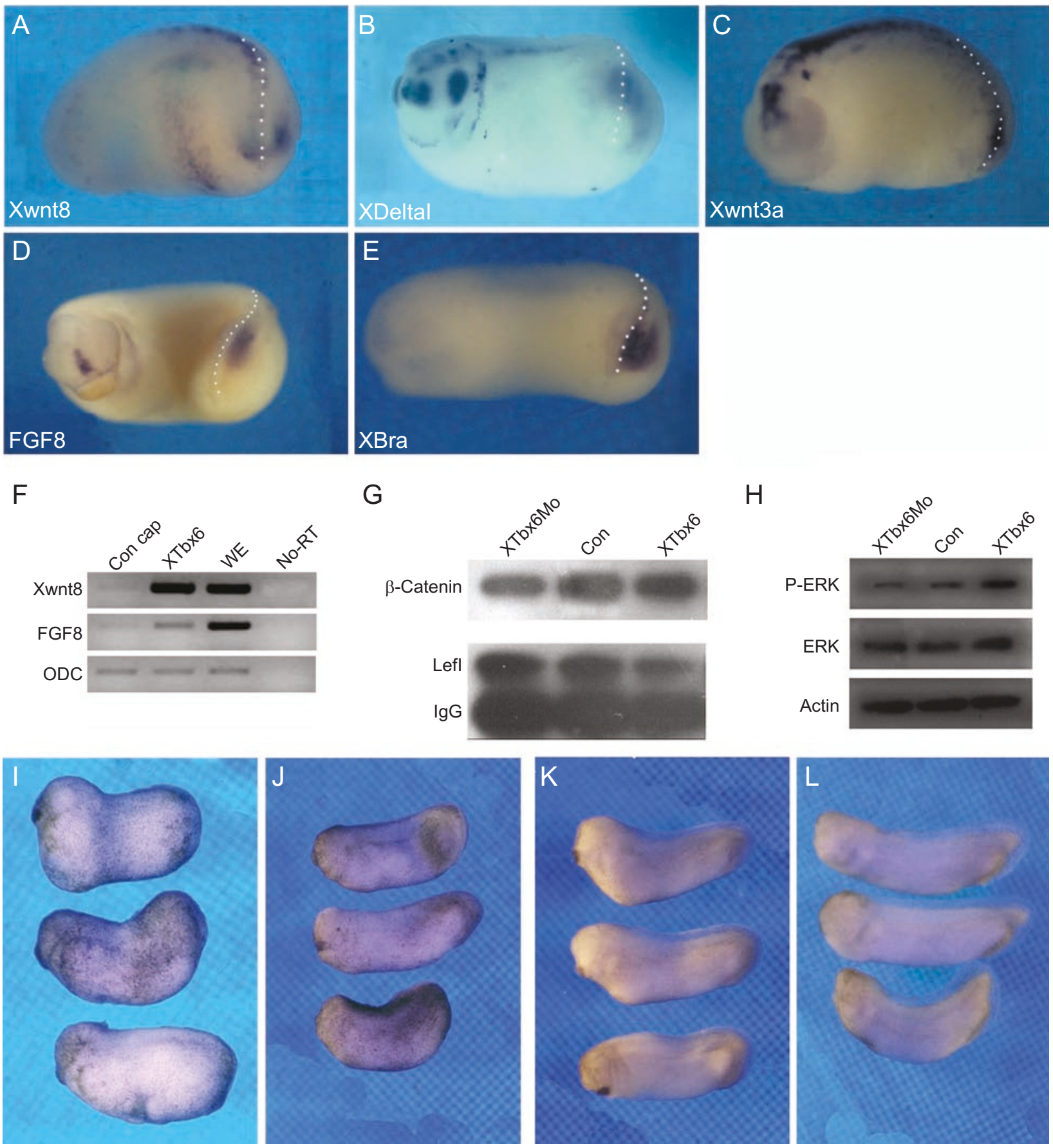

Figure 4 Xenopus Tbx6 mediates posterior patterning via Wnt and FGF signalling pathways. (A-E) Knockdown of $X T b x 6$ through injecting XTbx6Mo into one side of the ventral marginal zone at four-cell stage led to reduced expression of Xwnt8 (A), XDelta I (B), FGF8 (D) and Xbra (E) but not Xwnt3a (C). (A-E) Stage 23, lateral view, head to the left. The embryos bend to the side injected with XTbx6Mo, and the other side serves as the control. White dot lines indicate the midline of the embryos. (F) XTbx6 could induce $X w n t 8$ and FGF8. Con cap, control cap; WE, whole embryo at stage 12. NO-RT, no reverse transcriptase control with whole embryo total RNA; ODC, ornithine decarboxylase as the loading control. (G) XTbx6 could influence nuclear localization of $\beta$-catenin. Lane 1: Embryos were injected with XTbx6Mo+Lef-1-HA; lane 2: embryos were injected with Lef-1-HA only; and lane 3: embryos were injected with XTbx6 mRNA+Lef-1-HA. IgG represents the loading control. (H) XTbx6 could influence phosphorylation of ERK. Lane 1: Embryos were injected with XTbx6Mo; lane 2: control embryos; and lane 3: embryos were injected with XTbx6 mRNA. P-ERK: phosphorylated ERK. Actin serves as the loading control. (I-L) Antagonists of Wnt or FGF signalling pathway could block the induction ability of XTbx6. (I) Embryos were injected with Lef-1-Engrailed mRNA. (J) Embryos were injected with XTbx6 plasmid and Lef-1-Engrailed mRNA. (K) Embryos were injected with XFD mRNA. (L) Embryos were injected with $X T b x 6$ plasmid and XFD mRNA. (I-L) Stage 25, lateral view, head to the left. 
into ventral marginal zone at four-cell stage (Figure 4A and 4D), with $X w n t 3 a$ not affected (Figure 4C). Importantly, $X$ DeltaI and $X b r a$, two important downstream effectors of Wnt and FGF signalling pathways, respectively [35-37], also showed marked downregulation (Figure 4B and 4E).

To confirm whether the XTbx6-induced expression of Wnt and FGF ligands indeed leads to activation of these two signalling pathways in vivo, we checked the amount of the nuclear localized $\beta$-catenin and phosphorylation of ERK, which indicate the activation of these two respective pathways. Result from co-immunoprecipitation between injected HA-tagged Lef- 1 and endogenous $\beta$-catenin showed that the amount of nuclear localized $\beta$-catenin in XTbx 6 mRNA-loaded embryos was higher than that in Lef-1 injected alone, and the opposite phenomena appeared in XTbx6Mo-loaded embryos (compared with control embryos) (Figure 4G). ERK phosphorylation is an indication of FGF signalling pathway activation. Western blot with the antibody specifically recognizing phosphorylated ERK protein showed that the amount of phosphorylated ERK protein was upregulated in the embryos loaded with XTbx 6 mRNA, and was downregulated in the embryos loaded with XTbx6Mo, compared with control embryos (Figure 4H).

To further prove that FGF and Wnt signalling mediate $X T b x 6$ function, Wnt signalling antagonist Lef-1-Engrailed mRNA or FGF signalling antagonist XFD mRNA was coinjected with $X T b x 6$ (Figure 4J and 4L) into dorsal animal pore at four-cell stage. Lef-1-Engrailed is a fusion protein consisting of the full-length Lef-1 and the transcriptional repressor domain of the Drosophila Engrailed protein added to C-terminal of Lef-1 protein. Lef-1 binds to the same DNA sequence as Tcf-3 [38], and overexpressed Lef-1-Engrailed efficiently represses Wnt target genes in Xenopus (our unpublished results and reference [39]). In both cases (46/54 with XTbx 6 and Lef-1-Engrailed, 43/50 with XTbx 6 and XFD), the effect of XTbx6 induction was largely blocked. Taken together, our results demonstrate that XTbx6 plays a crucial role in posterior patterning through modulating endogenous FGF and Wnt signalling pathways.

\section{Discussion}

The patterning of the vertebrate posterior body has been investigated in mouse, avian, fish and amphibian embryos. Accumulating evidence indicated the existence of a tail organizer, which, like the head organizer, plays key roles in patterning the posterior body structures. Molecular mechanism involved in organizer activity has been studied and signalling pathways like FGF and Nodal are demonstrated to be the tail organizer components. However, there is still much remaining unknown about this complicated process, especially about the interaction of signalling pathways.

Tbx6 is believed to be involved in vertebrate posterior body development. In Tbx6 null mouse, the differentiation of paraxial mesoderm was profoundly affected, with the posterior paraxial tissue differentiating into neural fate instead of forming somites, and the formation of posterior body was also largely impaired [18]. This dramatic alteration indicates that mouse $T b x 6$ is needed for cells adopting the paraxial mesoderm cell fate, and needed to pattern the posterior body structure as well. However, the multiple morphological defects in Tbx6 knockout mouse make it difficult to identify how XTbx6 functions in different developmental events. Our data presented here provide solid in vivo evidence that $X T b x 6$ is sufficient and required for the formation of posterior structures in Xenopus embryos. The XTbx6-induced ectopic posterior structure displays a perfect morphological and histological pattern. We have also transplanted XTbx6-loaded animal tissue into stage 13 neural plate, to test the induction capacity in a similar assay as that performed by Beck and Slack [4], and found that the animal tissue was indeed able to induce posterior structure similar to that induced by injection of XTbx 6 into animal pole (data not shown). Furthermore, we found that the initiation of posterior structure by introducing $X T b x 6$ into embryos could only be achieved by injecting DNA into the dorsal animal pole, whose descendants are destined to neural ectoderm, but not the other part of the embryos. Injecting mRNA was less effective in terms of producing the ectopic posterior structure. Since the previous reports have indicated that interaction between neural ectoderm and mesoderm is important for development of the tail [4, 5], the evidence we presented here provides valuable cues for further investigation about how Tbx6 exerts its function in posterior patterning.

The finding that $X T b x 6$ is sufficient and required for the formation of posterior structures in Xenopus embryos (Figures 1 and 2) also allows us to incorporate $X T$ Tbx 6 into the signalling network of regulators for the posterior body formation. There is a substantial set of data demonstrating that the Wnt signalling pathway is an essential component of the posterior organizing center in vertebrate embryos [ 40 , 41], and members of the FGF family are thought to play important roles in trunk and tail formation [14]. Brachyury, another T-box transcription factor, which can engage in mutual activation with FGF and maintains a positive-feedback loop [36], is also essential for the development of the posterior part of the embryo [42]. In this current work, we identified $W n t 8$ and $F G F 8$ as downstream targets of $X T b x 6$, and manipulation of $X T b x 6$ level in embryos could strongly affect the endogenous activities of Wnt or FGF signalling pathways (Figure 3). As XTbx6 is regulated by BMP, FGF 


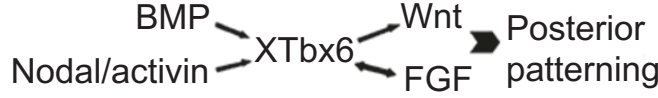

Figure 5 The model of $X T b x 6$-mediated posterior patterning. Multiple signalling pathways such as BMP, FGF and activin/Nodal signalling can regulate $X T b x 6$, and it could influence FGF and Wnt signalling pathways during posterior patterning.

and Nodal/activin signalling pathways [19], it is thus reasonable to position $X T b x 6$ in the molecular network, which controls the posterior development in Xenopus (Figure 5). For further investigation, experiments should be carried out to examine how the positive-feedback loop between FGF and XTbx6 is maintained and determine the direct target genes activated by XTbx6 (XWnt8, FGF 8 and $X b r a$ all are candidates). These efforts will help us to understand how the molecular network is synchronized during embryo posterior patterning.

In Tbx6 null mouse, three neural tubes form at the expense of the posterior somites [18] and the Tbx6 null ES cells fail to populate posterior somites in chimeric embryos [43]. It is worthy to perform lineage-tracing experiments to identify the cell fate of $X T b x 6$-loaded cells in the induced posterior structure. Results from this experiment could drop a hint to us that XTbx6 might direct the mesoderm differentiation in a cell-autonomous manner, and promote ectoderm patterning in a non-cell-autonomous way.

During the preparation of this manuscript, Li et al.[44] reported that $F G F 8, X w n t 8$ and $X M y f 5$ are target genes of $X T b x 6$, and that these target genes act downstream and mediate the function of $X T b x 6$ in anteroposterior specification. Our results are consistent with this study, which together with our in vivo loss-of-function and gain-of-function experiments provided convincing evidence to the conclusion that $X T b x 6$ mediates posterior patterning via regulation of Wnt and FGF signalling.

\section{Acknowledgments}

We thank Dr Peter S Klein (Department of Medicine and Cell and Developmental Biology, University of Pennsylvania), Dr Christof Niehrs (Division of Molecular Embryology German Cancer Research Center) for constructs, and Dr Schneider (Max-Planck-Institut fur Entwicklungsbiologie) for b-catenin antibody. We also thank Dr Jinhu Wang and Dr Zhao He for discussion of the project and critical reading of the manuscript. This work was supported by the National Natural Science Foundation of China (90408005, 30270650) and the National Key Project for Basic Science Research of China (2001CB509901).

\section{References}

1 Niehrs C. Regionally specific induction by the Spemann-Mangold organizer. Nat Rev Genet 2004; 5:425-434.

2 Holmdahl DE. Experimentelle Untersuchungen über die Lage der Grenze zwischen primärer und sekundärer Körperentwicklung beim Huhn. Anat Anz 1925; 59:393-396.

3 Tucker S. The Xenopus laevis tail-forming region. Development 1995; 121:249-262.

4 Beck CW, Slack JM. A developmental pathway controlling outgrowth of the Xenopus tail bud. Development 1999; 126:16111620.

5 Beck CW, Whitman M, Slack JM. The role of BMP signaling in outgrowth and patterning of the Xenopus tail bud. Dev Biol 2001; 238:303-314.

6 Beck CW, Slack JM. Notch is required for outgrowth of the Xenopus tail bud. Int J Dev Biol 2002; 46:255-258.

7 Takada S, Stark KL, Shea MJ, Vassileva G, McMahon JA, McMahon AP. Wnt-3a regulates somite and tailbud formation in the mouse embryo. Genes Dev 1994; 8:174-189.

8 Kelly GM, Greenstein P, Erezyilmaz DF, Moon RT. Zebrafish wnt8 and wnt8b share a common activity but are involved in distinct developmental pathways. Development 1995; 121:17871799.

9 Rauch GJ, Hammerschmidt M, Blader P, et al. Wnt5 is required for tail formation in the zebrafish embryo. Cold Spring Harb Symp Quant Biol 1997; 62:227-234.

10 Liu P, Wakamiya M, Shea MJ, Albrecht U, Behringer RR, Bradley A. Requirement for Wnt3 in vertebrate axis formation. Nat Genet 1999; 22:361-365.

11 Aulehla A, Wehrle C, Brand-Saberi B, et al. Wnt3a plays a major role in the segmentation clock controlling somitogenesis. Dev Cell 2003; 4:395-406.

12 Kishida M, Koyama S, Kishida S, et al. Axin prevents Wnt-3ainduced accumulation of beta-catenin. Oncogene 1999; 18:979985.

13 Shum AS, Poon LL, Tang WW, et al. Retinoic acid induces downregulation of Wnt-3a, apoptosis and diversion of tail bud cells to a neural fate in the mouse embryo. Mech Dev 1999; 84:17-30.

14 Amaya E, Musci TJ, Kirschner MW. Expression of a dominant negative mutant of the FGF receptor disrupts mesoderm formation in Xenopus embryos. Cell 1991; 66:257-270.

15 Amaya E, Stein PA, Musci TJ, Kirschner MW. FGF signalling in the early specification of mesoderm in Xenopus. Development 1993; 118:477-487.

16 Pownall ME, Tucker AS, Slack JM, Isaacs HV. eFGF, Xcad3 and Hox genes form a molecular pathway that establishes the anteroposterior axis in Xenopus. Development 1996; 122:38813892.

17 Mathis L, Kulesa PM, Fraser SE. FGF receptor signalling is required to maintain neural progenitors during Hensen's node progression. Nat Cell Biol 2001; 3:559-566.

18 Chapman DL, Papaioannou VE. Three neural tubes in mouse embryos with mutations in the T-box gene Tbx6. Nature 1998; 391:695-697.

19 Fang PF, Hu RY, He XY, Ding XY. Multiple signaling pathways control Tbx6 expression during Xenopus myogenesis. Acta Biochim Biophys Sin 2004; 36:390-396.

20 Nieukoop PD, Faber J. Normal Table of Xenopus Laevis. New 
York: Garland Publishing, 1994.

21 Christen B, Slack JM. FGF-8 is associated with anteroposterior patterning and limb regeneration in Xenopus. Dev Biol 1997; 192:455-466.

22 Henrique D, Adam J, Myat A, Chitnis A, Lewis J, Ish-Horowicz D. Expression of a Delta homologue in prospective neurons in the chick. Nature 1995; 375:787-790.

23 Sharpe CR, Fritz A, De Robertis EM, Gurdon JB. A homeoboxcontaining marker of posterior neural differentiation shows the importance of predetermination in neural induction. Cell 1987; 50:749-758.

24 Ekker SC, McGrew LL, Lai CJ, et al. Distinct expression and shared activities of members of the hedgehog gene family of Xenopus laevis. Development 1995; 121:2337-2347.

25 Harland RM. In situ hybridization: an improved whole-mount method for Xenopus embryos. Methods Cell Biol 1991; 36:685695.

26 Ruiz i Altaba A. Planar and vertical signals in the induction and patterning of the Xenopus nervous system. Development 1992; 116:67-80.

27 Kintner CR, Brockes JP. Monoclonal antibodies identify blastemal cells derived from dedifferentiating limb regeneration. Nature 1984; 308:67-69.

28 Klymkowsky MW, Hanken J. Whole-mount staining of Xenopus and other vertebrates. Methods Cell Biol 1991; 36:419-441.

29 Agius E, Oelgeschlager M, Wessely O, Kemp C, De Robertis EM. Endodermal Nodal-related signals and mesoderm induction in Xenopus. Development 2000; 127:1173-1183.

30 Kofron M, Demel T, Xanthos J, et al. Mesoderm induction in Xenopus is a zygotic event regulated by maternal VegT via TGFbeta growth factors. Development 1999; 126:5759-5770.

31 Steinbeisser H, Fainsod A, Niehrs C, Sasai Y, De Robertis EM. The role of gsc and BMP-4 in dorsal-ventral patterning of the marginal zone in Xenopus: a loss-of-function study using antisense RNA. EMBO J 1995; 14:5230-5243.

32 He Z, Li J, Zhen C, Feng L, Ding X. Knockdown of p53 by RNAi in ES cells facilitates RA-induced differentiation into muscle cells. Biochem Biophys Res Commun 2005; 335:676-683.

33 Uchiyama H, Kobayashi T, Yamashita A, Ohno S, Yabe S. Clon- ing and characterization of the T-box gene Tbx6 in Xenopus laevis. Dev Growth Differ 2001; 43:657-669.

34 Chapman DL, Agulnik I, Hancock S, Silver LM, Papaioannou VE. Tbx6, a mouse T-Box gene implicated in paraxial mesoderm formation at gastrulation. Dev Biol 1996; 180:534-542.

35 Yamaguchi TP, Takada S, Yoshikawa Y, Wu N, McMahon AP. T (Brachyury) is a direct target of Wnt3a during paraxial mesoderm specification. Genes Dev 1999; 13:3185-3190.

36 Isaacs HV, Pownall ME, Slack JM. eFGF regulates Xbra expression during Xenopus gastrulation. EMBO J 1994; 13:44694481.

37 Hofmann M, Schuster-Gossler K, Watabe-Rudolph M, Aulehla A, Herrmann BG, Gossler A. WNT signaling, in synergy with T/TBX6, controls Notch signaling by regulating D111 expression in the presomitic mesoderm of mouse embryos. Genes Dev 2004; 18:2712-2717.

38 Eastman Q, Grosschedl R. Regulation of LEF-1/TCF transcription factors by Wnt and other signals. Curr Opin Cell Biol 1999; 11:233-240.

39 Geng X, Xiao L, Lin GF, et al. Lef/Tcf-dependent Wnt/betacatenin signaling during Xenopus axis specification. FEBS Lett 2003; 547:1-6.

40 Yamaguchi TP. Heads or tails: Wnts and anterior-posterior patterning. Curr Biol 2001; 11:R713-R724.

41 Holland LZ. Heads or tails? Amphioxus and the evolution of anterior-posterior patterning in deuterostomes. Dev Biol 2002; 241:209-228.

42 Conlon FL, Sedgwick SG, Weston KM, Smith JC. Inhibition of Xbra transcription activation causes defects in mesodermal patterning and reveals autoregulation of Xbra in dorsal mesoderm. Development 1996; 122:2427-2435.

43 Chapman DL, Cooper-Morgan A, Harrelson Z, Papaioannou VE. Critical role for Tbx6 in mesoderm specification in the mouse embryo. Mech Dev 2003; 120:837-847.

44 Li HY, Bourdelas A, Carron C, Gomez C, Boucaut JC, Shi DL. FGF8, Wnt8 and Myf5 are target genes of Tbx6 during anteroposterior specification in Xenopus embryo. Dev Biol 2006; 290:470-481. 